\title{
Innovación y transferencia: la implementación del hallazgo
}

\section{Knowledge transfer and innovation: discovery implementation}

\author{
Dr. Eng. Juan R. Castillo Molina \\ Coordinador de innovación Arquitectónica \\ Escuela de Arquitectura y Urbanismo, Universidad Nacional Pedro Henríquez Ureña (UNPHU). \\ Santo Domingo, República Dominicana
}

Fecha de recepción: 31 de marzo de 2019

Fecha de aceptación: 6 de mayo de 2019

Favor citar este artículo de la siguiente forma:

Castillo Molina, JR. (2019). Innovación y transferencia: la implementación del hallazgo.

AULA Revista de Humanidades y Ciencias Sociales. Vol. 64. Número 4, enero-junio 2019. Santo Domingo: Amigo del Hogar.

http://dx.doi.org/2636.2236/AULA.2019.005

\section{RESUMEN}

La humanidad ha archivado investigaciones inéditas que raras veces impactan la práctica de las profesiones. El presente artículo explora cómo la implementación de hallazgos académicos depende de la transferencia de conocimiento interdisciplinario para ir más allá del plano teórico. Se analiza el concepto de innovación a través de la introducción de campos que usualmente no se debaten en las escuelas de arquitectura (cósmico, energético, cognitivo, molecular y financiero), y se enfatiza sobre la importancia de la recuperación y revitalización de los inventos latentes. Como resultado, se propone la implementación del hallazgo a través de un proceso de desplazamiento, amplificación y validación de resultados que tengan el potencial de formular nuevas rutas académicas y profesionales, y finalmente detonen el reinicio de la profesión.

Palabras clave: amplificación, código, hallazgos, inteligencia artificial, invención.

\begin{abstract}
Humanity has stored significant inventions that rarely impact the general public. The present paper explores how the implementation of academic findings excel theoretical fields, and how knowledge transfer is significant for its application. The paper conceptualizes on innovation through discussing fields usually ignored in architectural schools (cosmic, cognitive, energetic, molecular and economic) and includes the importance of recovery and revitalization of latent inventions. As a result, the paper proposes the implementation of discoveries through the process of displacing, amplifying and validating the results that could lead to new academic and professional trends, and finally reboot the whole profession.
\end{abstract}

Keywords: Artificial intelligence, coding, discoveries, invention, upgrading. 


\section{Introducción}

Las instituciones educativas poseen hallazgos que permanecen ocultos del saber genérico, aunque virtualmente estén al alcance de toda la sociedad en sus bases de datos. La humanidad ha archivado investigaciones para uso posterior, pero es necesario que se implementen los inventos contenidos en ellas para formular nuevas rutas académicas y oportunidades profesionales. El conocimiento aplicable es aquel que puede ser utilizado inmediatamente u optimizado para implementarse a corto plazo, pues la aplicación de los mismos produce nuevos conocimientos como respuesta, dándole la oportunidad de que se conviertan en nuevos descubrimientos.

La implementación es el proceso ideal para transferir conocimiento de la academia a la práctica, especialmente cuando el hallazgo se aprovecha y se convierte en innovación. Esta dinámica ocurre algunas veces en el campo empírico (práctica), y otras veces sucede en un ambiente controlado (academia). Tradicionalmente, el invento se implementa para validar nuevas ideas, asegurando la evolución de la práctica como soporte de la innovación.

Usualmente, el invento permanece en el mundo académico generando valor cultural. Los hallazgos están regularmente en estado investigativo o publicados en medios especializados para ser discutidos mayormente por eruditos, evitando que sus aportes tengan el impacto esperado en el plano práctico. Los experimentos cuestionan el status quo, y hoy tienen la oportunidad de expandirse a medida que la inteligencia computacional eficientiza la comunicación entre profesiones disímiles. Aunque la transferencia de conocimiento exige ajustes, pues explorar una idea en un ambiente hipotético es distinto a implementarla bajo condiciones reales, la manera en que se está combinando lo aprendido (análogo) con lo avanzado (digital) es una oportunidad para evolucionar la práctica arquitectónica convencional. La implementación aporta valores más allá de lo cultural, produciendo beneficios en el campo socio-económico.
La práctica arquitectónica obedece a la inmediatez, pues se concentra en la respuesta eficiente validada por la costo-efectividad, fungiendo como artificio donde las hipótesis se encuentran con la realidad empírica y eliminando hallazgos que representen riesgos económicos. Simultáneamente, la misma puede ser un recurso importante para llevar experimentos más allá del plano teórico, pues a través de las oportunidades de realización que ella facilita, los descubrimientos teóricos se pueden convertir en innovaciones al alcance de todos. Aunque parezca difícil implementar hallazgos, la práctica profesional se mantiene en la búsqueda de nuevas ideas, abriendo oportunidades de aplicación para las proposiciones académicas. Igualmente, la práctica hace descubrimientos empíricos que deben ser estudiados en el plano académico para reducir la distancia entre pensamiento y acción.

El presente ensayo plantea que el verdadero valor de los hallazgos académicos va más allá del plano teórico, pues su utilidad reside en su aplicación práctica. La arquitectura es una industria del conocimiento, y como tal, debe contribuir directamente implementando descubrimientos para lograr transformaciones más efectivas de las ciudades.

\section{Innovación: evidencia precisa}

La innovación es una evidencia de la evolución humana. Tradicionalmente, ha sido plano de debates científicos y religiosos, abriendo preguntas sobre orígenes y revoluciones teóricas tratando de encontrar una justificación a la existencia. Sin embargo, al estar dominada por la inteligencia humana, avanza sigilosamente, haciendo mejoras y actualizaciones que tienen un impacto infalible en la protección de la especie, proyectando vías hacia la sofisticación del planeta.

La innovación deja evidencias tangibles, así se mide y se expande; puede reusarse o reinterpretarse por culturas disímiles pasando por un proceso de adaptación inevitable, pero antes de llegar a esa fase, permanece incógnita 
o escondida hasta que se hace latente y se descubre. Su valor va más allá del económico, pues depende de la perpetuidad de la humanidad. La invención demanda flexibilidad absoluta, desarrollándose en campos no lineales, que proponen reajustes de acuerdo a las necesidades de implementación.

La innovación carece de tiempos. Aunque el futuro está asociado a invenciones, estas últimas obedecen a una necesidad universal y no a la inmediatez de un tiempo que resulta incierto bajo las evoluciones tecnológicas a la que es sometida la humanidad. La innovación posee campos de acción para actualizar el conocimiento aplicable: cósmico, energético, cognitivo, molecular y financiero. Se debe indagar en los trabajos científicos y artísticos que tocan esos campos para encontrar el modo de aplicación que ayudará a la profesión a manejar los retos socioeconómicos, culturales y medioambientales evidentes en las ciudades.

\section{Campo cósmico}

El campo cósmico es aquel que estudia el espacio exterior para alcanzar la expansión de la humanidad hacia el universo. La falta de gravedad en la travesía o el aumento de la misma en el planetizaje, las bajas temperaturas en el trayecto o altas en el destino planetario, los cambios de presión en el despegue o aterrizaje y demás condiciones extraterrestres aumentan el riesgo de las estructuras por un lado, pero ofrecen oportunidades infinitas de invención para sus arquitectos.

La relación de la arquitectura con el diseño aeroespacial es histórica. Desde la primera década del siglo pasado las escuelas soviéticas proyectaron ciudades flotantes tratando lo que hoy se conoce como diseño aeroespacial (Magomedov, 2015). Con el descubrimiento del cohete, la llamada carrera espacial utilizó la ciencia ficción para plantear hábitats capaces de sobrevivir condiciones hostiles extraterrestres. Comenzaron a surgir estructuras de todas las escalas desde estaciones, colonias mineras, hasta llegar a megaestructuras que suministraban todo el confort de un planeta (Asimov, 1974). Se impulsó un nuevo horizonte ficticio que ha ayudado a dar forma a estructuras terrícolas destinadas a zonas climáticas extremas.

La Base Halley VI es una estación científica británica localizada en la Antártica cuyo diseño utiliza soluciones tecnológicas derivadas de la industria aeroespacial. Las bajas temperaturas del contexto exigieron que el espesor de las paredes, piso y techo se manufacture por capas de elementos aislantes, terminando exteriormente en una resina que aumente la vida útil de la estructura metálica interior (Halley VI, 2013). El método constructivo usó la prefabricación, que aunque no es exclusivo del diseño aeroespacial, ha sido validado por el mismo. El presupuesto de la estación es alto comparado con una estructura erigida en otra parte del planeta, pero eso no impide considerar al campo cósmico como una fuente de innovación arquitectónica.

El cine ha fortalecido la relación entre arquitectura y diseño aeroespacial. La estética de la película 2001: Odisea Espacial (Stanley Kubrick, 1968) ha sido el paradigma arquitectónico de una estructura espacial, aunque la misma fue derivada de los proyectos oficiales de la NASA por medio de ilustraciones (Agel, 1970). Hoy día, se piensa en ambientes «futuristas» como soluciones basadas en un aspecto impecable, con líneas precisas que funden la funcionalidad y apariencia del objeto, derivadas de la sofisticación expuesta en esa película.

\section{Campo energético}

El campo energético es útil para indagar conocimiento aplicable, pues es difícil imaginar arquitectura carente de fuentes energéticas. La sostenibilidad ha explotado el tema energético hacia críticas que favorecen las fuentes renovables, alzando argumentos de alerta sobre las soluciones nucleares y petroleras. Las fuentes renovables exigen una revisión total del diseño arquitectónico, desde su conceptualización hasta su proceso de trabajo. La combinación 
absoluta de tecnología y forma arquitectónica es tradicional en arquitectura, y lo sostenible la revisita como campo para reinventar la imagen final de los proyectos. La oportunidad de innovación radica en entender cómo el sistema eléctrico se modificará, posiblemente reduciendo su propia infraestructura para pasar a una totalidad inalámbrica; esto puede producir un beneficio para el costo total de la obra y un aumentar el confort interior si se combina con inteligencia artificial.

Cada vez más se está llegando a una fusión entre practicidad y apariencia de los elementos constructivos, como el panel solar transparente que funciona como cierre y fuente energética simultáneamente; estas ideas pueden devenir en una arquitectura donde cada parte sea un artificio polifuncional. La arquitectura del presente siglo debe indagar en investigaciones de ese tipo, para reforzar esa relación indispensable entre ente arquitectónico y energía, y proponer hacia cuáles direcciones puede dirigirse la profesión tras la aplicación de conocimientos aparentemente ajenos a la misma.

\section{Campo cognitivo}

La innovación en el campo cognitivo maneja el conocimiento por medios presenciales o virtuales. Esta área presenta cuatro pasos condicionados a la calidad y cantidad de información manipulada: producción, almacenaje, accesibilidad y difusión. La producción es una habilidad humana, donde cada actividad genera datos infinitos casi imposible de reutilizar, salvo por la labor de las universidades que exploran, seleccionan y sistematizan el saber humano convirtiéndolo en producto de dominio público. La práctica profesional también produce contenido difícil de almacenar debido a que su récord es la obra construida, a sabiendas que a la Arquitectura le interesa todo hallazgo que pueda aplicarse en áreas tecnológicas e intelectuales, independientemente si fue producido en la práctica o la academia. Los formatos digitales han agilizado el almacenamiento, ya que todo se comprime en datos que pueden recuperarse a través de la interfase que conecta el usuario con el contenido. La accesibilidad a la fuente es inmediata usando el internet, causando que métodos de investigación tradicionales se autoevalúen debido a que la red posee casi todo archivo humano, haciendo que las primeras fases se agilicen (recopilación de datos, por ejemplo).

El conocimiento exige arqueología para rastrear y validar la fuente y poder difundirse. La discusión de un libro es un ejemplo concreto de difusión pues sus datos formulan ideas con posible aplicación más allá del objetivo mismo del texto. La Arquitectura se ha enriquecido reinterpretando contenidos formulados en otras áreas, y adaptándolos a una filosofía estética que garantice la permanencia del legado humano. (Le Corbusier, 1923)

La inteligencia artificial (IA) es imprescindible para navegar en el campo cognitivo, y aunque se haya visualizado a través de la robótica, el campo central de la misma es la programación. Replicar la mente humana es complicado pues todavía no se ha llegado a la potencia exponencial de la naturaleza, y la ciencia está tomando otra ruta donde lo que se está optimizando es el ser humano en sí y no la máquina, logrando amplificar el cuerpo para llegar a tener superinteligencia (Wilson, 2013). Es posible que en condiciones posthumanas la obtención y manejo de datos llegue a ser una capacidad incluida en el cuerpo, y que los implantes trabajen como antena de búsqueda y aplicación de información conectados a otras fuentes artificiales de producción, almacenamiento y difusión del conocimiento. (Kurtzweil, 2005)

\section{Campo molecular}

El campo molecular explora la invención de nuevos materiales de construcción. Originalmente, los avances se determinaban empíricamente hasta que la ciencia de materiales formalizó y utilizó la simulación virtual en ese campo. Esta área permanece parcialmente investigada por la Arquitectura, demorando su aplicación en una estructura 
total. La costo-efectividad de los proyectos controla la implementación de tecnología de nueva generación, evitando que exista un edificio hecho completamente de materiales avanzados y limitándolos a elementos de la fachada y piezas de diseño interior. La creación de ambientes inmersivos (realidad virtual) explora el comportamiento de nuevos materiales constructivos, haciendo que las pruebas digitales simulen un edificio en su totalidad desde generalidades de funcionamiento hasta la capacidad molecular de un componente específico. Las ciencias médicas están desarrollando la nanotecnología para disminuir el tamaño de los objetos que estén en contacto directo con el cuerpo humano, facilitar la aceptación del dispositivo implantado y eliminar los efectos secundarios del ente extraño. Ese proceso también ha llegado a la arquitectura en forma de compuestos y metamateriales, pero su aplicación está en proceso (Dunn, 2012). La singularidad propone artificios que aumentan el uso de espacios virtuales, reduciendo el tamaño del espacio arquitectónico y aumentando el paisaje de esparcimiento.

El campo molecular debe ser implementado en la práctica arquitectónica para conseguir la reducción del tamaño de los objetos, disminuyendo el uso de materias primas, entendiendo que la evolución no depende de una aceleración tecnológica sino de los descubrimientos que salvaguarden el planeta. El académico puede identificar inventos moleculares, y planificar junto a los diseñadores de materiales la manufactura y ensamblaje de elementos constructivos.

\section{Campo financiero}

El campo financiero es donde se analiza la visión del arquitecto junto a todas las partidas del proyecto para alcanzar la materialización de la obra. Las investigaciones académicas arquitectónicas raras veces exploran la costo-efectividad de las invenciones, y es necesario formar un equipo de planificadores, arquitectos, financieros, ingenieros y diseñadores de materiales para llevar a cabo una propuesta económica precisa y avanzada. Los programas computacionales facilitan la comunicación cruzada entre disciplinas disímiles. Dentro de la ciencia de la informática, toda información es reducida a un código binario que permite que los diferentes datos naveguen indistintamente a su traducción final en cada disciplina (Casey, 2010). El BIM (modelado de información de construcción o building information modeling) transforma gráficos en dígitos numéricos que a su vez pueden transferirse hacia los softwares de todos los involucrados en el diseño. Inversamente, la entrada numérica afecta la propuesta arquitectónica, asegurando un intercambio cibernético libre de subjetividades.

Los descubrimientos inéditos producidos a través de los campos cósmico, energético, cognitivo, molecular y financiero son evidencias infalibles si se consideran como conocimiento aplicable en vez de experimentos teóricos. Las escuelas de arquitectura deben dejar de considerar la innovación como una exploración abierta y verla como una posibilidad precisa, de forma que las tesis y proyectos de investigación hagan énfasis en la implementación de hallazgos inéditos y garanticen que las academias ayuden a mejorar el estilo de vida cambiante de la humanidad.

\section{Hallazgo: naturaleza extendida}

Los hallazgos son una consecuencia de la evolución de las civilizaciones. Usualmente, los inventos se ven ajenos a las estructuras orgánicas y minerales terrestres, pero son elementos procesados e implementados por la inteligencia humana, una poderosa herramienta físico-química, que a la vez es una extensión de la naturaleza.

\section{Recuperación de hallazgos históricos}

La recuperación de hallazgos para uso ulterior es una tradición de la inteligencia humana. La arquitectura de finales del siglo XX utilizó los descubrimientos de la vanguardia rusa (Constructivismo/Suprematismo) para crear nuevo conocimiento (Libeskind, 1992). 
Las ideologías de diseño encontradas produjeron una sucesión de nuevos estilos, conectando ideas inéditas centenarias con conceptos contemporáneos, validando la importancia del descubrimiento académico para la aplicación profesional. La máquina, una vez entendida a través de su relación propósito/apariencia, continúa siendo protagónica pero esta vez transformada en el código como unidad de programación digital.

A la velocidad que van introduciéndose novedades, es difícil establecer un significado claro de lo que representa ser histórico, pero el concepto historia se actualiza constantemente acorde a la tecnología. El uso del dispositivo LIDAR (Light Imaging Detection and Ranging) ha contradicho argumentos que se daban como verdades, como el caso de los mohaís de la Isla de Pascua considerados anteriormente como cabezas solamente y hoy día se sabe que el resto del cuerpo está enterrado en el suelo; es decir, la historia ha pasado de ser un contenedor de hechos a un campo de probabilidades. De manera inversa, la velocidad comercial introduce artilugios que amenazan en convertir en obsoleto todo aquello fuera de la categoría de datos digitales (Rams, 2012). Por ejemplo, la música siempre ha estado avanzada en relación con los instrumentos que la producen, provocando que un nuevo aparato sea inventado para revelar el último experimento acústico. Tradicionalmente, los diferentes canales de audio pertenecían al mundo sónico interno del autor, y no fueron evidentes para el público hasta la aparición del sonido estereofónico, aunque ha habido una vuelta a la reproducción análoga de la música (cintas y vinilo) con la certeza de que esos medios tienen un ancho de banda ilimitado, contrario a la grabación digital que posee uno fijado al momento de realizarla, aunque el primer medio pierda su valor sónico debido a la frecuencia de uso. Es necesario que en todas las profesiones se recuperen los hallazgos históricos archivados para disminuir el tiempo y esfuerzo de producir e implementar nuevos conocimientos.

\section{Revitalización del hallazgo latente}

El hallazgo latente debe revitalizarse a través de las Ciencias y las Humanidades. La tecnología es un logro significativo de la humanidad, al parecer todo puede alcanzarse utilizándola. La Física y la Química la estructuran usando el cálculo matemático como eje transversal. La lógica se encarga de darle sentido a los resultados y buscarle aplicación exitosa, pero el algoritmo, código de control computacional de toda tecnología contemporánea, está abierto a la manipulación y obstrucción del funcionamiento original del artificio. «En el mundo computacional, un algoritmo es una secuencia de instrucciones que, procesadas, deviene un listado de opciones para resolver problemas de diseño. Es un proceso tripartito donde primero el diseñador introduce y codifica los datos necesarios (input), segundo, el programa ofrece una serie ilimitada de respuestas (output), y tercero, el arquitecto escoge la opción óptima para formalizar su respuesta. Es decir, el arquitecto, cuyo rol tradicional era encontrar soluciones basadas en conocimientos finitos, es ahora transformado en un agente sensible que toma decisiones basadas en una gama infinita de posibilidades programadas») (Castillo y Arismendy, 2017, p.1). Además, la tecnología tiene su propia velocidad de invención más allá de las necesidades humanas, pues los artilugios con retornos económicos menores se convierten en obsoletos aunque tengan mayor potencial que los que existen en el mercado. Esto debe motivar a que se revisen los archivos universitarios, y se recuperen de allí inventos inéditos que impulsen nuevos descubrimientos arquitectónicos.

El hallazgo latente es tecnológico e intelectual, y esta última cualidad es la que completa la Arquitectura como profesión que explora Ciencia y Humanidades por igual. Los resultados se convierten en descubrimientos intelectuales que inspiran nuevos inventos a través del pensamiento crítico. Las formulaciones teóricas de una tesis arquitectónica son tan relevantes 
como el resultado proyectual, pues se pueden analizar por separado y obtener conclusiones distintas y válidas a la vez. Esa libertad de exploración debe ejercerse en la academia, pues la práctica contiene riesgos económicos y prejuicios culturales que entorpecerían el proceso.

Hay que evitar usar la arqueología del pasado para imponer modelos únicos que fomenten el rechazo de soluciones innovadoras. La revitalización del hallazgo no significa rechazar la invención de nuevos inventos, al contrario, se trata de revisar lo que existe, analizarlo sistemáticamente y descubrir cómo llegar al próximo nivel de desarrollo profesional.

\section{Implementación: más allá del código}

La implementación de los hallazgos va más allá del código cuando se conectan perfectamente la programación computacional con la inteligencia humana. Esa dinámica ha venido ocurriendo lentamente desde hace décadas y su proceso va evolucionando hacia la integración de funciones entre máquina y organismo (Gibson, 1984). El código binario está aumentando la velocidad de procesamiento de la información, al punto que es imposible pensar en implementación de nuevos hallazgos sin considerar la informática como un recurso de cálculo avanzado.

La introducción de la intuición humana en los procesos informáticos va más allá del código. El algoritmo todavía no ha logrado trabajar aislado, ya que necesita los insumos intuitivos del diseñador. Aunque los resultados computacionales pueden llegar a ser infinitos, las definiciones algorítmicas son limitadas, pues no pueden ir más allá de lo que fueron programadas al depender de los datos específicos insertados (Asimov, 1955). Los hallazgos evolucionan si salen fuera de los límites definidos por los objetivos y alcances de las tesis por ser marcos de referencia que están diseñados para transgredirse en futuras exploraciones. Los programas de investigación que utilicen la inteligencia artificial pueden actualizarse a través de un proceso de desplazamiento, amplificación y validación.

\section{Desplazamiento}

El desplazamiento sucede cuando el objetivo teórico se desplaza hacia el propósito práctico. En este caso, la acción es conceptual y se enfoca en el análisis y sustitución de los insumos inoperantes. Es una manera de comprobar cuáles aspectos de la investigación deben tomarse en cuenta si se desea implementar un hallazgo. Es un razonamiento que se concentra en mecanismos proactivos, como la manera de alertar sobre datos inaceptables o peligrosos que puedan cuestionar los objetivos y alcances de las propuestas. Además, es un proceso perfecto para que la transferencia de conocimiento invite a experimentar fuera del campo académico. El desplazamiento no es aleatorio, pues la lógica de sus autores (orgánicos y artificiales) selecciona qué parte de la investigación será desplazada. El proceso puede utilizar conceptos que sitúen el asunto bajo estudio en un contexto inimaginable dando paso al cuestionamiento del hallazgo original.

El desplazamiento implica dirección y trayectoria. Mover el conocimiento hacia otro campo necesita una serie de líneas de acción, algunas conectadas directamente, otras a través de intermediarios y otras encontradas por la experimentación, pero todos los vectores se concretizan en la lógica humana y artificial del software. La fuga hacia otros campos implica que el desplazamiento sucede en un contexto limitado y usualmente ajeno al inicial. Los límites son cruciales para indicar precisamente lo que se debe transgredir, marcando hacia cuáles fronteras el concepto se trasladará.

El desplazamiento también incluye la capacidad de comunicar conceptos que no son inmediatamente percibidos. Al manejarse en un modo conceptual, las ideas seleccionadas a desplazarse son abstractas y es precisamente la abstracción donde la inteligencia humana y la 
artificial se conectan, logrando que el objetivo teórico se desplace al propósito práctico.

\section{Amplificación}

La amplificación de los recursos de exploración exige entender el funcionamiento de las herramientas de investigación. Las metodologías científicas son las más comunes, aunque las más recientes están utilizando los programas computacionales que están introduciendo inteligencia artificial para conectar disciplinas disímiles. Los cálculos complejos invitan a que se estudie la estructura informática del software para entrar en la ingeniería del mismo y utilizarlo experimentalmente. La educación en programación provee estrategias para burlar el código, mejorando la relación entre máquina y usuario, y exigiendo del último un aumento en su capacidad natural de procesamiento de datos.

La dependencia en cómputo asistido comenzó con el uso de calculadoras de funciones integrales y ha pasado a una interfase donde la fórmula queda oculta, transformada en una ventana de comandos, e instaurando un lenguaje universal basado en la relación software/usuario perteneciente a la Tercera Revolución Industrial (3IR). La implementación de la inteligencia artificial ha logrado que el rendimiento computacional sea dominado por la máquina, liberando al usuario de horas infinitas de razonamiento matemático, y permitiéndole incurrir en actividades intuitivas que solo él puede lograr hasta ahora y que complementan las precisiones de la computadora. Recurrir a la ayuda inmediata de la computación artificial es una costumbre que va en aumento, estableciéndola como la herramienta para alcanzar una amplificación ilimitada (Rodríguez y Castillo, 2019).

\section{Validación}

La validación de los resultados no la determina el software sino la inteligencia humana. El código está diseñado para ocultar información que sería abrumadora para el proceso de razonamiento tradicional, aumentando la dependencia de artilugios digitales, que asumidos al extremo, podrían limitar el desarrollo de la mente. La máquina es una forma sofisticada de procesamiento de datos, pero aún es inferior a la subjetividad experimental del cerebro como herramienta del descubrimiento y el asombro. La neurociencia ha comprobado que el cerebro humano tiene la capacidad de aumentar su velocidad de pensamiento, contrario al poco éxito que han tenido los esfuerzos de replicar las funciones de la mente, ya que demanda un tiempo de investigación tan infinito como su sujeto (Kurzweil, 2005). La implementación usando solamente el ingenio avanzado es una opción válida, aunque por el momento se recurra a la ayuda de la computación artificial como recurso inmediato; es decir, el desarrollo de la inteligencia humana al máximo podría plantear la abstinencia del uso de la computadora, una evolución que la ciencia todavía necesita completar para inaugurar la posibilidad de ir más allá del código.

Validar un resultado es un oficio de toda actividad humana, especialmente para aquellas que trabajan con ciencia y estética. La ciencia tiene sus canales de aprobación claros, pero la estética posee la imaginación como habilidad de autenticación (Virilio, 1988). La arquitectura utiliza ambas, pues se apoya en tecnología de próxima generación y en argumentos teóricos de vanguardia para proceder con la validación de propuestas inéditas, especialmente las académicas.

La validación de los resultados va más allá del código al concentrar sus esfuerzos científicos con los aspectos humanísticos que sustentan la creación de las máquinas. La artificialidad es un recurso significativo para las civilizaciones por su poder de suplir necesidades cruciales y debido a que su evolución ha tenido un trayecto que hoy se conoce bajo el concepto de lo exponencial, por lo tanto, llegará a un nivel donde sea tan imprescindible como los elementos naturales que hacen posible los ecosistemas. $\mathrm{La}$ relación artilugio/invento es obligatoria en la invención, y solo a través de la transmisión de 
conocimiento comprobado con la implementación de los hallazgos latentes en las academias la innovación tendrá un sentido que vaya más allá de la acumulación de datos potenciales que nunca se aplicarán.

\section{Reinicio: nueva profesión}

Las investigaciones inéditas deben plantear un reinicio o un nuevo comienzo de planteamientos y estrategias basadas en conocimiento aplicable. Los procesos utilizados desde el siglo pasado han demostrado ser ineficientes, pues a la producción en masa y la customización avanzada les ha tocado surgir en una época que ha protegido la sobreproducción de objetos a favor de la continua desaparición del patrimonio planetario; además, ambas son soluciones intermedias entre una edad de desastres antropomórficos y un status quo forzado por una economía mundial desbalanceada. Reiniciar la profesión no trata de institucionalizar rebeldías vanguardistas, sino de revisar lo inoperante, transformar lo que obstruye el progreso de la humanidad e inaugurar una era que supere el concepto mismo de civilización.

La relación entre Arquitectura y naturaleza debe entenderse más allá de soluciones de sostenibilidad. La naturaleza debe ser un campo de inspiración pues tiene resueltos muchos problemas que la Arquitectura todavía no ha podido abordar, pero la arquitectura es un asunto artificial y debe ser considerado como tal para optimizar su relación con la naturaleza. Ambas comparten lugares adyacentes en el entorno, y la arquitectura debe ser más eficiente en conseguir salvaguardar la naturaleza. En esta, la evolución, cambios o revoluciones toman siglos, mientras en el mundo humano ese fenómeno toma solamente décadas. Esa es una gran diferencia, y por eso la naturaleza se renueva a sí misma casi infinitamente. Los humanos no pueden renovar nada sin destruirlo, aunque tienen al reciclaje como un logro significativo que palidece frente a lo que la naturaleza alcanza. Las academias necesitan entender la artificialidad de la arquitectura, pues cada elemento que utiliza proviene de recursos naturales limitados y debe seguir produciendo materiales sintéticos que reduzcan la extracción desmedida de las materias primas; el producto final siempre será un cuerpo extraño insertado en el entorno, pues ya no puede estar hecho totalmente de materiales orgánicos por razones medioambientales.

La Arquitectura maneja la ciencia y las humanidades, y como tal, debe liderar el desarrollo de proyectos, pero necesita reiniciarse. La Arquitectura ha demostrado que resulta efectiva al momento de tener una visión de conjunto objetiva y sensible al medioambiente, pues está formada para analizar todas las variables científicas y artísticas de la obra.

Como la Arquitectura abarca los campos de la ciencia y las humanidades, sus métodos de investigación deben ser una combinación de lo científico con lo argumentativo, y no uno de los dos específicamente; igualmente, debe evitarse usar un formato de publicación fijo, pues necesita ser de acuerdo a la naturaleza y objetivo de la investigación arquitectónica, y en caso que se necesite fijar uno, es necesario crearlo tras una comparación sistemática de los existentes.

Los inventos archivados en instituciones académicas son la base para un nuevo discurso arquitectónico, pues posiblemente las soluciones a problemáticas contemporáneas ya están en esas investigaciones latentes. Contrario a lo que el desarrollo inmobiliario ha hecho desde el siglo pasado, la Arquitectura sabe dominar la visión de conjunto, optimizando lo interdisciplinario, evitando la sobreproducción de objetos y potencializando la inclusión de todo conocimiento no-lucrativo que beneficie al mundo. La profesión debe reinventar sistemas financieros, técnicos y estéticos que permitan alcanzar otras fronteras, y debe hacerlo a través de la implementación de los hallazgos latentes en las academias para continuar la tradición humana de amplificar la tecnología de próxima generación junto a la naturaleza milenaria que siempre existirá más allá de la humanidad. 


\section{Referencias}

Agel, J. (1970). The making of Kubrick's 2001. New York, NY: Signet, Signet Classics.

Anderson, R. (2013). Ludwig Hilberseimer: Metropolis Architecture and Selected Essays. New York, NY: GSAPP BOOKS.

Asimov, I. (1957). The Naked Sun. Garden City, New York: Doubleday \& Company, Inc.

Asimov, I. (1974). Our World in Space. Greenwich, Conn: New York Graphic Society, Ltd.,

Bozzola, A. (1977). Futurism. New York, NY: Penguin Books.

Casey, R. (2010). Form + Code in Design, Art and Architecture. New York, NY: Princeton Architectural Press.

Castillo, J. \& Arismendy, J. (2017). Algoritmo generativo. Santo Domingo. Recuperado de http://docentes.unibe. edu.do/algoritmo-generativo/

Denari, N. (1999). Gyroscopic Horizons. New York, NY: Princeton Architectural Press.

Dunn, N. (2012). Proyecto y construcción digital en arquitectura. Barcelona: Art Blume, S.L.

Gibson, W. (1984). Neuromante. Barcelona: Ediciones Minotauro.

Halley VI British Antarctic Research Station. (2013). A Pioneerign Relocatable Polar Science Research Station. Recuperado de https://hbarchitects.co.uk/halley-vi-british-antarctic-research-station/

Hobsbawm, E. (1999). A la Zaga. Decadencia y fracaso de las vanguardias del siglo XX. Barcelona: Crítica, S.L.
Jullier, L. (1998). La imagen digital: de la tecnología a la estética. Buenos Aires, Argentina: La Marca.

Kurzweil, R. (2005). The singularity is near. New York, NY: Penguin Books.

Le Corbusier.(1923). Vers Une Architecture. Paris: Flammarion.

Levene, R. (1995). Zaha Hadid. 1988-1991. Barcelona: El Croquis.

Libesbind, D. (1992). Countersign. New York, NY: Rizzoli International Publications, Inc.

Magomedov, S. (2011). Heroes of the Avant-Garde. Ivan Leonidov. Moscow: M., Sergey Gordeev.

Magomedov, S. (2015). Georgii Krukitov: the Flying City and beyond. Moscow: Tevnov Books.

Miyake, I. (2012). Pleats Please. Koln: Taschen.

Rams, D. (2014). Less But Better. Hamburg: Jo Klatt Design+Design Verlag.

Rand, A. (1996). Atlas Shrugged. New York, NY: Signet.

Richter, H. (1997). Dada Art And Anti Art. New York, NY: Thames \& Hudson, Inc.,

Rodriguez, K. \& Castillo, J. (2019). Inteligencia artificial y su aplicación en un proceso de diseño validado. Santo Domingo: UNPHU.

Rubio, O. (2014). El Lissitzky. The Experience of Totality. Madrid: La Fábrica.

Suzuki, A. (2001). Do Android Crows Fly Over the Skies of an Electronic Tokyo?. London: AA Publications.

Virilio, P. (1988). La estética de la desaparición. Barcelona: Editorial Anagrama, S.A.

Wilson, D. (2013). Amped. New York, NY: Vintage Books.

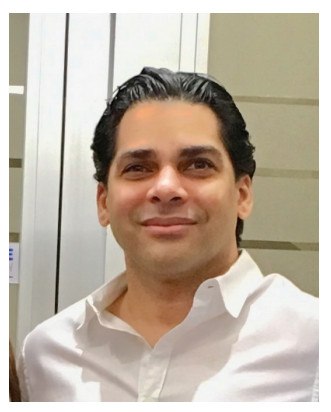

\section{Juan R. Castillo}

Arquitecto y docente interesado en procesos avanzados arquitectónicos. Egresado de Arquitectura (UNPHU, 1994), obtuvo Doctorado en Hiroshima University (Japón, 2002), jurado de proyectos de maestría en Columbia University (USA, 2010) y es profesor titular en la Universidad Iberoamericana (UNIBE). Ha publicado seis artículos en el Journal of Architecture, Planning and Environmental Engineering AIJ (Tokyo), Misawa Bauhaus Collection (Tokyo) y Portal Docente UNIBE. Estuvo asociado a Shearly Investments, Inc., produciendo proyectos turísticos, habitacionales e industriales. Actualmente es miembro del Architectural Institute of Japan (AIJ), CODIA y SARD, jurado del concurso ADOACERO y practica desde su firma FUTURA, Arquitectura Emergente. 